\title{
ANAlysis Of ACOUSTIC CHANNEL CHARACTERISTICS FOR UNDERWATER WIRELESS SENSOR NETWORKS
}

\author{
Yamini Kularia ${ }^{1}$, Sheena Kohli ${ }^{2}$ and Partha Pratim Bhattacharya ${ }^{3}$ \\ ${ }^{1}$ Department of ECE, College of Engineering and Technology, Mody University of \\ Science and Technology, Lakshmangarh, India \\ ${ }^{2}$ Department of CSE, College of Engineering and Technology, Mody University of \\ Science and Technology, Lakshmangarh, India \\ ${ }^{3}$ Department of ECE, College of Engineering and Technology Mody University of \\ Science and Technology, Lakshmangarh, India
}

\begin{abstract}
Underwater Wireless Sensor Networks (UWSNs) find numerous applications, like underwater monitoring \& exploration, water quality analysis, offshore oil field monitoring, oceanographic data collection etc. All these aquatic applications need to observe \& predict the ocean characteristics. The conventional methods used for terrestrial domain cannot be applied here as there exist some architectural differences in the underwater environment, mainly due to variations in the transmission medium. In this paper, we have analyzed the acoustic channel characteristics like attenuation, noise and speed of sound (propagation speed) with variations in frequency, depth, salinity, temperature etc. by applying different models and equations for UWSNs.
\end{abstract}

\section{KEYWORDS}

Underwater Wireless Sensor Network, Architecture, Attenuation profiles, Noise, Salinity, Sound Speed

\section{INTRODUCTION TO UNDERWATER WIRELESS SENSOR NETWORKS}

A Wireless Sensor Network (WSN) consists of a web of small sensor nodes, deployed in a specific geographical region. Each node is programmed to sense or monitor data, process it and communicate it with other nodes, external base station or sink. The sensor networks find diverse applications in residential, medical, commercial, industrial and military division [1].

As depicted in Figure 1, the surface of earth is occupied largely by water, which provides a lot of opportunities for exploration \& research in this field [2], calling for the need of an underwater sensor network that can intensify the abilities to explore the ocean [3].

DOI : $10.5121 /$ ijcsitce. 2016.3202 


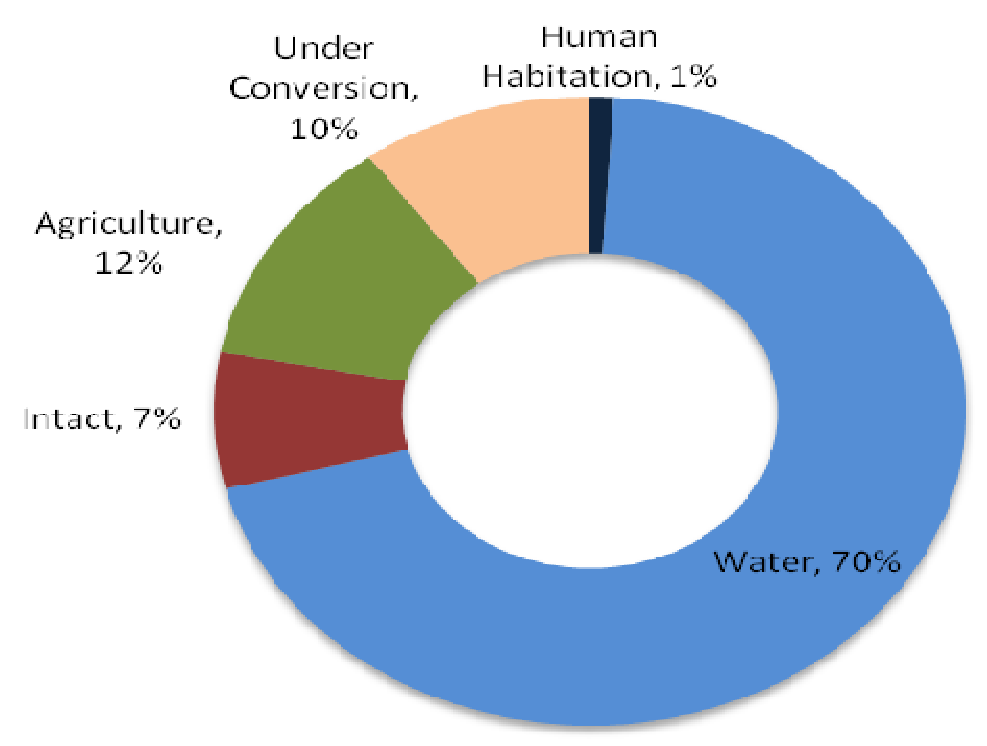

Figure 1. Water on the planet earth

Technically, both terrestrial and underwater WSNs operate on the same principle, but there are many differences between Underwater Wireless Sensor Networks (UWSNs) and the terrestrial ones. These differences are mainly because of the medium of transmission (sea water) and the signals used to transmit data (acoustic ultrasound signals). Most terrestrial methods fail to address the underwater applications [2]. Figure 2 shows the basic arrangement of Underwater Wireless Sensor Network environment.

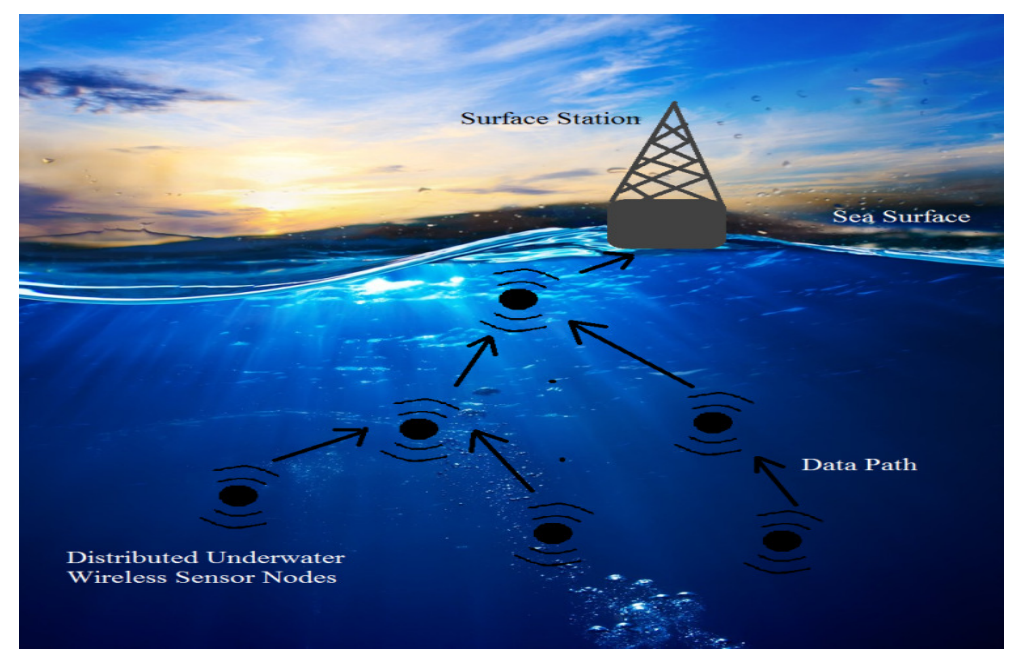

Figure 2. Underwater Wireless Sensor Network

For Underwater Wireless Sensor Networks, there are generally, two types of architectures: First is the static two-dimensional Underwater Acoustic Sensor Networks (UW-ASNs) for ocean bottom monitoring. A group of sensor nodes are anchored to the bottom of the ocean with anchors. 
These nodes are interconnected via wireless acoustic links forming clusters or groups, relaying data from the ocean bottom to the surface station, as shown in Figure 3. Another one is the threedimensional Underwater Acoustic Sensor Networks (UW-ASNs) used for ocean column monitoring. In this, the sensor nodes float at different depths in order to observe a given phenomenon as shown in Figure 4 [4].

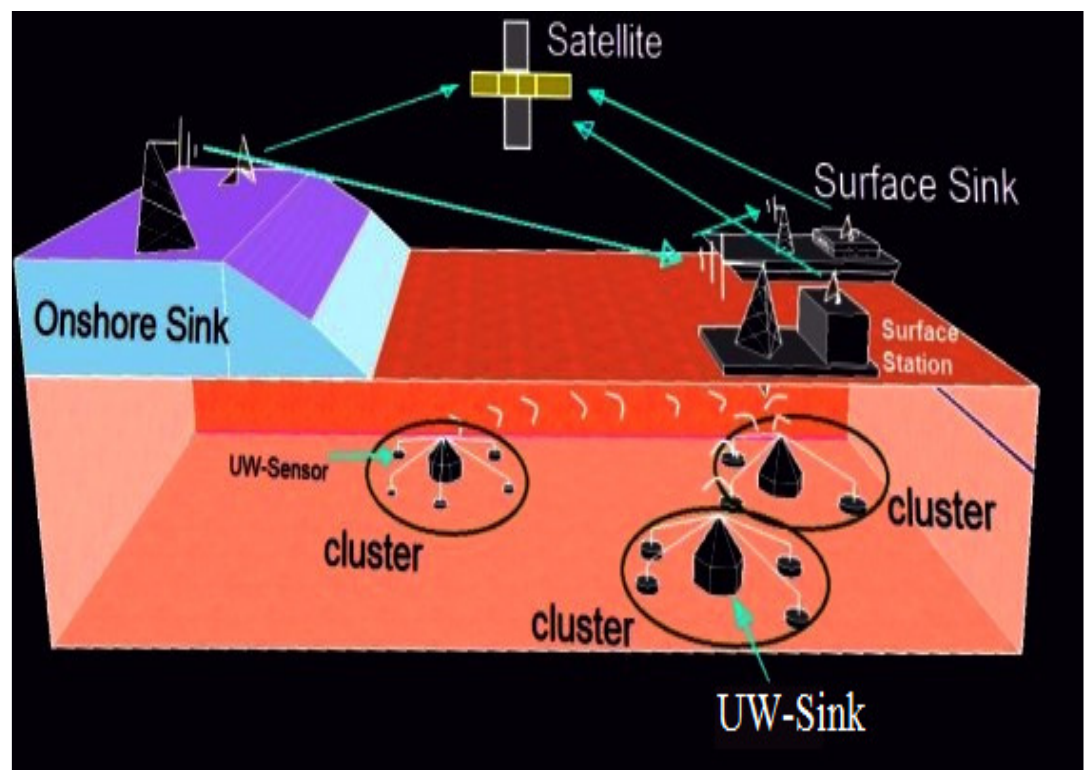

Figure 3. Architecture of 2D Underwater Sensor Networks

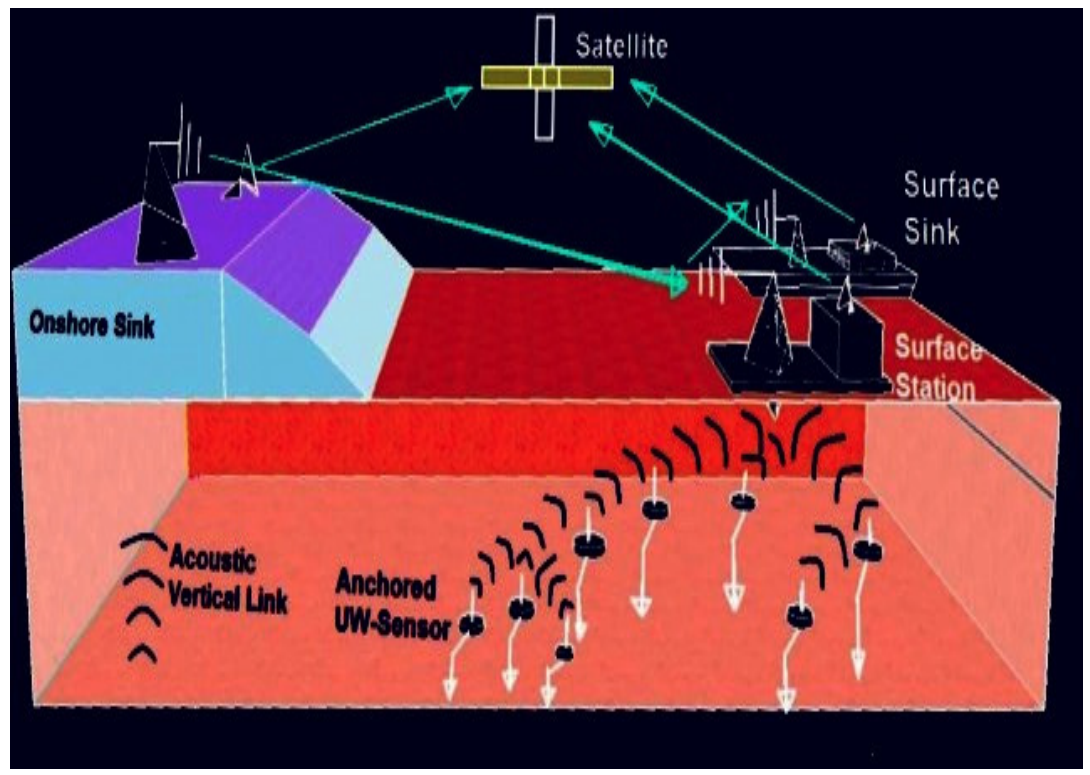

Figure 4. Architecture of 3D Underwater Sensor Network

UW-ASNs can perform pollution monitoring, monitoring of ocean's current and wind, detecting climate change, understanding and predicting the effect of human activity on marine ecosystems, 
tracking fishes or micro-organisms etc. The network may help detect underwater oilfields and valuable minerals, determine routes for undersea cables etc. Some underwater sensors that measure seismic activity can provide storm warnings to the coastal areas [5]. Sensors can be used to identify hazards on the seabed, locate dangerous rocks in water, mooring positions, submerged wrecks etc [6]. To make such applications feasible, acoustic channels need to be characterized. Using these channels is a major challenge. Certain identified problems in the communication system are signal attenuation, speed of sound and ambient noise in water.

The following work deals with the analysis and evaluation of the different communication constraints related to the special characteristics of water as a communication medium. The simulations have been done on MATLAB [7].

The remainder of the paper is organized as follows: Section 2 describes the acoustic models based on the fundamentals of underwater acoustic theory. Section 3 describes the implementation and the simulation results of the different characteristics and section 4 concludes the analysis for the same.

\section{Characterizing Acoustic Channel For Underwater Wireless SENSOR NETWORKS}

The medium of transmission is a critical factor for communication. Underwater wireless networking complicates the system. The following parameters must be studied in order to construct an accurate communication model:

\subsection{Attenuation}

Attenuation occurs due to the conversion of acoustic energy into heat. With seawater, the process of attenuation becomes frequency dependent. Energy absorbed by the water is proportional to the frequency of the signal. There are different equations describing the processes of acoustic attenuation in seawater, as discussed below in detail.

\subsubsection{Thorp Equation}

The Thorp model proposed in 1967 [8] involves the simplest equation for attenuation, taking into consideration the effect of the frequency utilized. It neglects the effect of frequencies caused by boric acid and magnesium sulphate, salinity and acidity levels of the water in sea or ocean, may not leading to a very accurate result. The Thorp Equation is formulated as:

$\alpha=0.11 \frac{f^{2}}{1+f^{2}}+44 \frac{f^{2}}{4100+f^{2}}+2.75 \times 10^{-4} f^{2}+0.003$ 
Here, $\mathrm{f}$ is frequency in $\mathrm{kHz}$

\subsubsection{Fisher \& Simmons Equation}

The Fisher \& Simmons model proposed in 1977 [9] is another most commonly used models. This model takes into account the effect of temperature and depth, for calculating the attenuation coefficient, introducing the effects of frequencies caused by boric acid and magnesium sulphate, which were ignored in the Thorp model. The Fisher \& Simmons Equation can be given as:

$\alpha=A_{1} P_{1} \frac{f_{1} f^{2}}{f_{1}^{2}+f^{2}}+A_{2} P_{2} \frac{f_{2} f^{2}}{f_{2}^{2}+f^{2}}+A_{3} P_{3} f^{2}$

Where, $A 1, A 2, A 3$ are functions of temperature and $P 1, P 2, P 3$ are functions of the constant equilibrium pressure. These are represented as:

$$
\begin{aligned}
& A_{1}=1.03 \times 10^{-8}+2.36 \times 10^{-10} T-5.22 \times 10^{-12} T^{2} \\
& A_{2}=5.62 \times 10^{-8}+7.52 \times 10^{-10} T \\
& A_{3}=\left[55.9-2.37 T+4.77 \times 10^{-2} T^{2}-3.48 \times 10^{-4} T^{3}\right] \times 10^{-15} \\
& f_{1}=1.32 \times 10^{3}(T+273.1) e^{\frac{-1700}{T+273.1}} \\
& f_{2}=1.55 \times 10^{7}(T+273.1) e^{\frac{-3052}{T+273.1}} \\
& P 1=1 \\
& P_{2}=1-10.3 \times 10^{-4} P+3.7 \times 10^{-7} P^{2} \\
& P_{3}=1-3.84 \times 10^{-4} P+7.57 \times 10^{-8} P^{2} \\
& P=D / 10
\end{aligned}
$$

Here, $f$ is frequency in $\mathrm{kHz}$

$\mathrm{T}$ is temperature in degrees Celsius

$\mathrm{D}$ is depth in meters

$f_{1}$ and $f_{2}$ are frequencies caused by boric acid and magnesium sulphate in $\mathrm{kHz}$

\subsubsection{Ainslie \& McColm Equation}

The Ainslie \& McColm equation proposed in 1998 [10] is based upon the Fisher \& Simmons model. However, it proposes some extra relaxation frequencies and simplifications to derive the following equation, hence increasing the applicability and probability of getting more accurate results:

$$
\alpha=0.106 \frac{f_{1} f^{2}}{f_{1}^{2}+f^{2}} e^{\frac{p H-8}{0.56}}+0.52\left(1+\frac{T}{43}\right)\left(\frac{S}{35}\right) \frac{f_{2} f^{2}}{f_{2}^{2}+f^{2}} e^{\frac{-D}{6}}+4.9 \times 10^{-4} f^{2} e^{-\left(\frac{T}{27}+\frac{D}{17}\right)}
$$

Here, $f$ is frequency in $\mathrm{kHz}$

$\mathrm{T}$ is temperature in degrees Celsius

$\mathrm{D}$ is depth in meters

$\mathrm{S}$ is salinity in parts per thousand

$f_{l}$ and $f_{2}$ are frequencies caused by boric acid and magnesium sulphate in $\mathrm{kHz}$ 
This McColm model also takes into account the effects of the $\mathrm{pH}$ of sea water. The equations for $f 1$ and $f 2$ are also simplified and represented in $\mathrm{kHz}$ :

$$
\begin{aligned}
& f_{1}=0.78 e^{\frac{T}{26}} \sqrt{\frac{S}{35}} \\
& f_{2}=42 e^{\frac{T}{17}}
\end{aligned}
$$

\subsection{Noise}

An important acoustic characteristic of UWSN is the underwater ambient noise. This could be regarding the state of sea surface, atmosphere, behaviour of marine animals etc. Ambient noise is made up of contributions from natural and manmade sources both. Sources like underwater explosion, blasting, machinery and shipping activities are contributors for manmade noise. Natural noise is related to hydrodynamics, seismic and biological phenomena. It comes from sources such as turbulence, wave noise, storms, rain, distant shipping etc [11]. Total noise for the acoustic channel is considered as a resultant of the following four factors [12]:

\subsubsection{Turbulence noise}

Turbulence associated with surface disturbance or tidal flow around an obstruction generates continuous noise.Turbulence is also caused by storms or during the rain events. It may be produced by marine life.

Turbulence noise is denoted by $N_{t}(f)$ in $\mathrm{dB}$ re micro $\mathrm{Pa}$ per $\mathrm{Hz}$ by:

$10 \log N_{t}(f)=17-30 \log (f)$

\subsubsection{Shipping Noise}

Another type of noise is the one caused by ship traffic. The effect of ship traffic is concerned with the number of ships and the distance of shipping from the area of study. It is denoted as $N_{s}(f)$

in $\mathrm{dB}$ re micro Pa per $\mathrm{Hz}$ (with $s$ as the shipping factor which lies between 0 and 1 for low and high activities respectively).

$10 \log N_{s}(f)=40+20(s-0.5)+26 \log (f)$

\subsubsection{Wave Noise}

Wave noise is caused due to the movement of waves in the sea or ocean. It is denoted as $N_{w}(f)$ in $\mathrm{dB}$ re micro Pa per $\mathrm{Hz}$ (with $w$ as the wind speed in $\mathrm{m} / \mathrm{s}$ ). The movement of the water results from tides, winds, currents and storms.

$10 \log N_{w}(f)=50+7.5 \sqrt{w}+20 \log (f)-40 \log (f+0.4)$ 


\subsubsection{Thermal Noise}

Thermal noise is denoted as $N_{t h}(f)$ in $\mathrm{dB}$ re micro $\mathrm{Pa}$ per $\mathrm{Hz}$, which can be taken as additive white Gaussian noise. Additive white Gaussian noise is the noise model used to mimic the effect of many random processes that occur in nature.Thermal noise is created by molecular agitation at the receiver side and it is always present in communication system.

$10 \log N_{\text {th }}(f)=-15+20 \log (f)$

\subsubsection{Total Noise}

The overall noise power spectral density for a given frequency $f$ can be computed by adding all types of noise as [12]:

$$
N(f)=N_{t}(f)+N_{s}(f)+N_{w}(f)+N_{t h}(f)
$$

\subsection{Sound Speed}

The prime method of wireless data communication in underwater is dependent on the acoustic medium and the most basic property affecting the data rate, quality of service, latency and other important network factors in the channel is the speed of sound. A sound wave can be considered as the mechanical energy that is transmitted by the source. A sound wave travels from one particle to another, being propagated through the ocean at the sound speed.

The propagation speed can be expressed by the following equation [13]:

$$
\begin{aligned}
& c=1449+4.6 T-5.304 \times 10^{-2} T^{2}+2.374 \times 10^{-4} T^{3}+1.340(S-35)+ \\
& 1.630 \times 10^{-2} D+1.675 \times 10^{-7} D^{2}-1.025 \times 10^{-2} T(S-35)-7.139 \times 10^{-13} D^{3}
\end{aligned}
$$

Where, $\mathrm{T}$ is temperature in degrees Celsius

$\mathrm{S}$ is salinity in parts per thousand

$\mathrm{D}$ is depth in meters

The approximate speed of sound in water is $1500 \mathrm{~m} / \mathrm{s}$, but varies between $1400 \leq \mathrm{v} \leq 1700 \mathrm{~m} / \mathrm{s}$. The Underwater Wireless Sensor Network is a complex environment that is affected by many varying factors, primarily temperature, salinity, depth and furthermore each of these factors may also be interdependent or may vary across the ocean at multiple locations and depths. Sensors in an underwater wireless network can be deployed at multiple depths, thereby encountering a range of temperatures as well. It is, thus, important to have an accurate model of the effects of these parameters on the speed of sound in water.

The salinity value for the ocean varies between $0.030 \mathrm{ppt}$ to $0.040 \mathrm{ppt}$, with a global depth and surface average of approximately 0.035 ppt. [14]. 


\section{RESUlTS AND ANALYSIS}

\subsection{Attenuation}

Attenuation has been computed by applying the equations 1 and 12. Figure 5 shows how attenuation changes with varying frequencies by using Thorp equation. Figure 6 shows how attenuation changes with varying frequencies using Ainslie \& McColm equation. For the simulation of these models, we consider Temperature $=30^{\circ} \mathrm{C}$, Salinity $=0.035 \mathrm{ppt}, \mathrm{pH}=8$ and Depth $=1000 \mathrm{~m}$. [15]

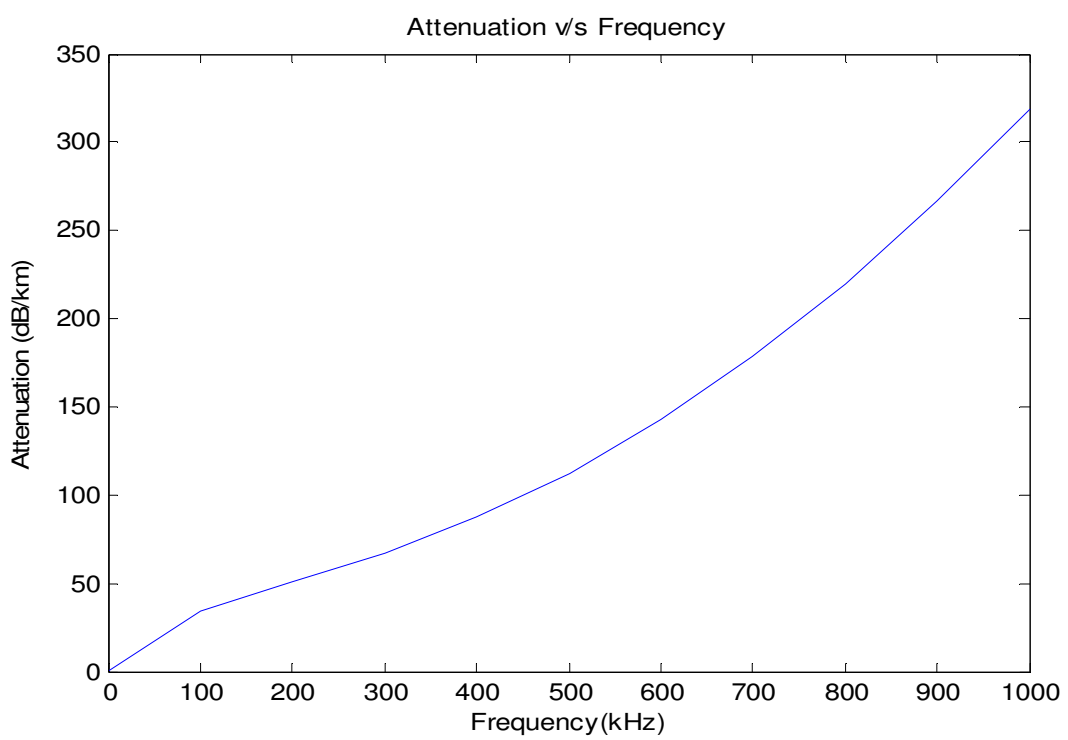

Figure 5. Attenuation coefficient with varying frequency using Thorp equation

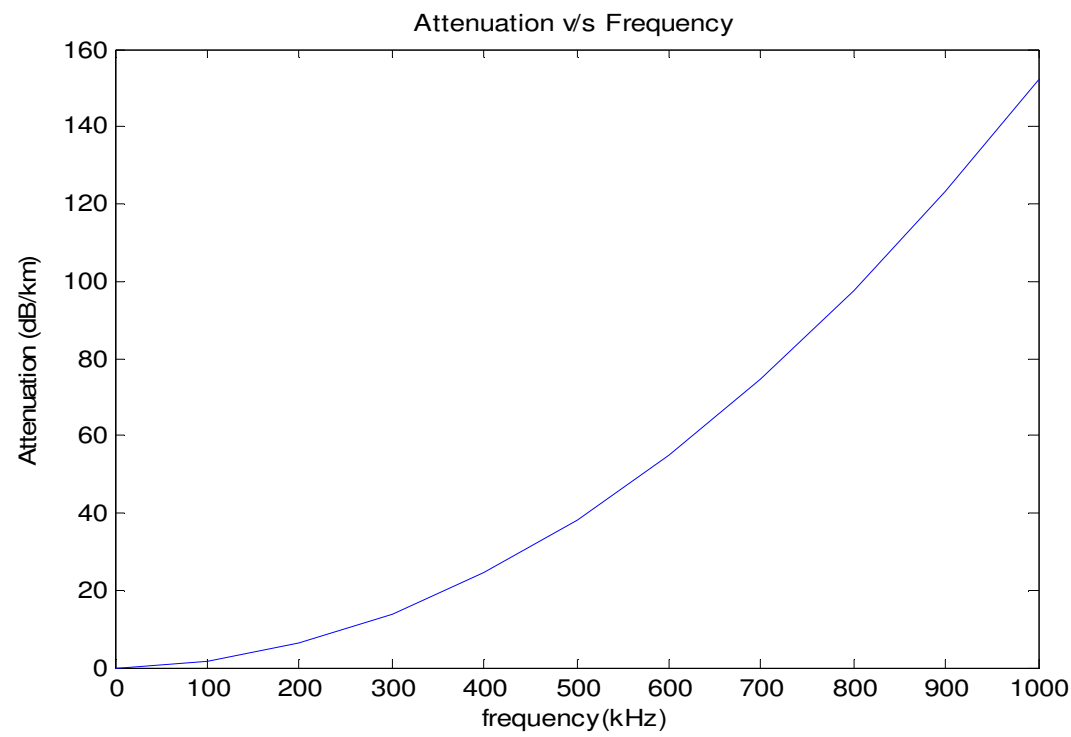

Figure 6. Attenuation coefficient with varying frequency and depth using Ainslie \& McColm equation 
From the simulation of attenuation models, we have observed that with increasing frequency and depth attenuation is also increased.

\subsection{Noise}

Noise has been computed from equation 19. Using this equation, results have been plotted in the graphs shown in Figures 7,8 and 9 with varying frequency $(10 \mathrm{~Hz}$ to $100 \mathrm{kHz})$, shipping factor ( 0.1 to 1$)$ and wind speed $(5 \mathrm{~m} / \mathrm{s}$ to $15 \mathrm{~m} / \mathrm{s})$ respectively [16]. Figure 7 shows the relation between noise and frequency with constant shipping factor $=0.5$, wind speed $=10 \mathrm{~m} / \mathrm{s}$ and varying frequency from $10 \mathrm{kHz}$ to $100 \mathrm{kHz}$. A larger frequency causes high noise in underwater.

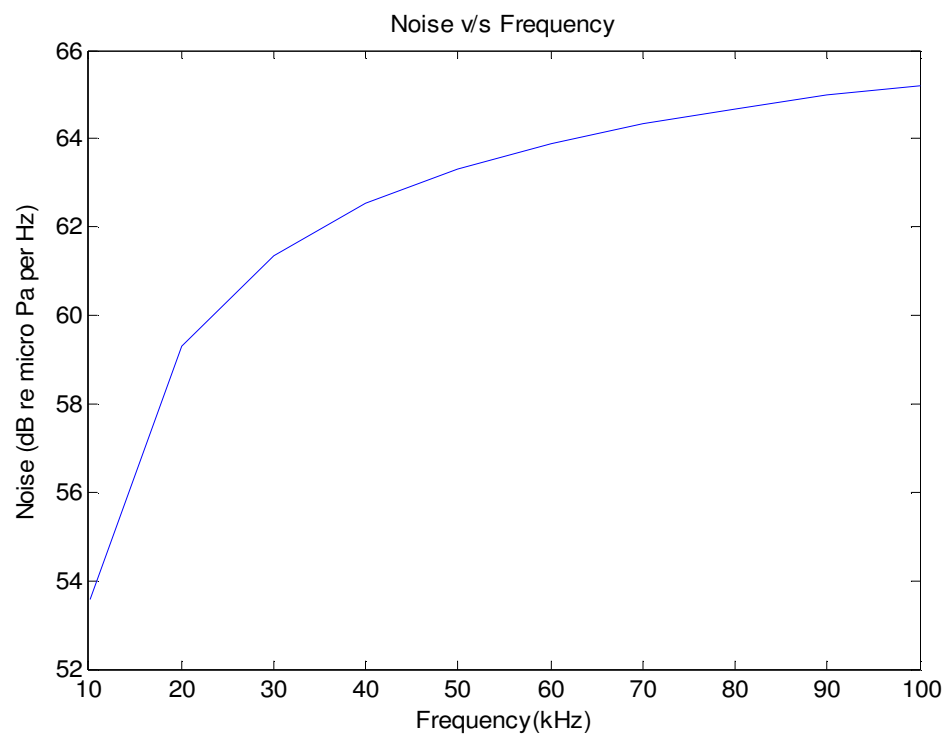

Figure 7. Noise v/s frequency

Figure 8 shows the relation between noise and shipping factor with constant frequency $=50 \mathrm{~Hz}$ (for shipping noise $10 \mathrm{~Hz}<\mathrm{f}<100 \mathrm{~Hz}$ ) [16], wind speed $=10 \mathrm{~m} / \mathrm{s}$ and varying shipping factor from 0.1 to 1 . The figure depicts that in underwater with increasing shipping factor, the noise increases. 
International Journal of Computational Science, Information Technology and Control Engineering (IJCSITCE) Vol.3, No.1/2, April 2016

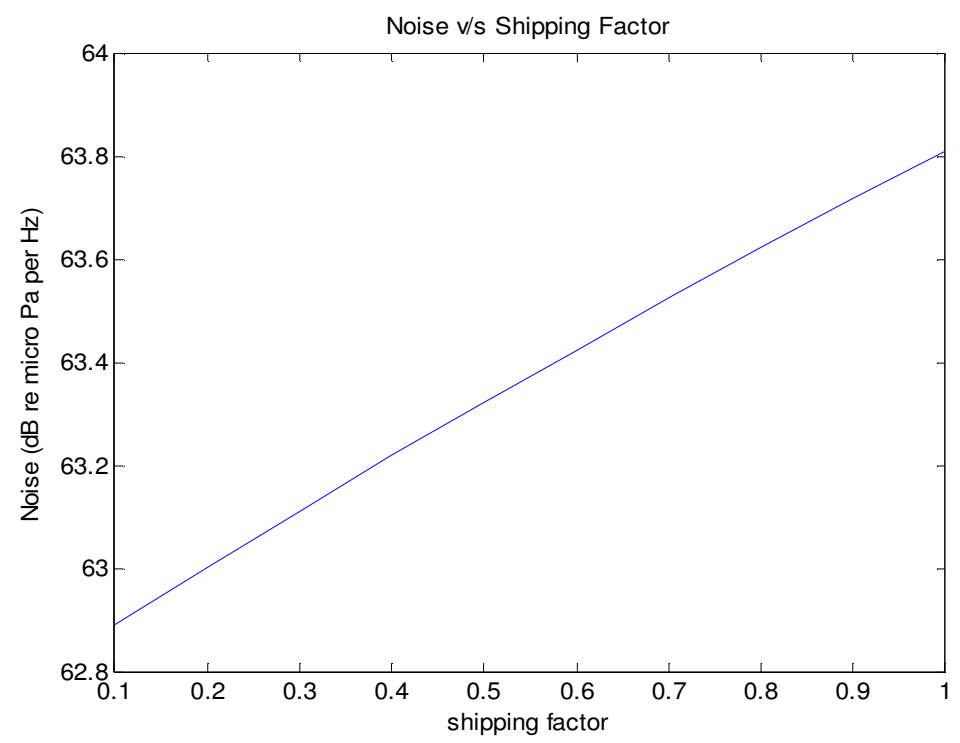

Figure 8. Noise v/s Shipping factor

Figure 9 shows the relation between noise and wind speed with constant frequency $=10 \mathrm{kHz}$ (for wind noise $100 \mathrm{~Hz}<\mathrm{f}<100 \mathrm{kHz}$ ) [16] and shipping factor=0.5 with varying wind speed from $5 \mathrm{~m} / \mathrm{s}$ to $15 \mathrm{~m} / \mathrm{s}$. With the increasing wind speed in underwater, noise also increases.

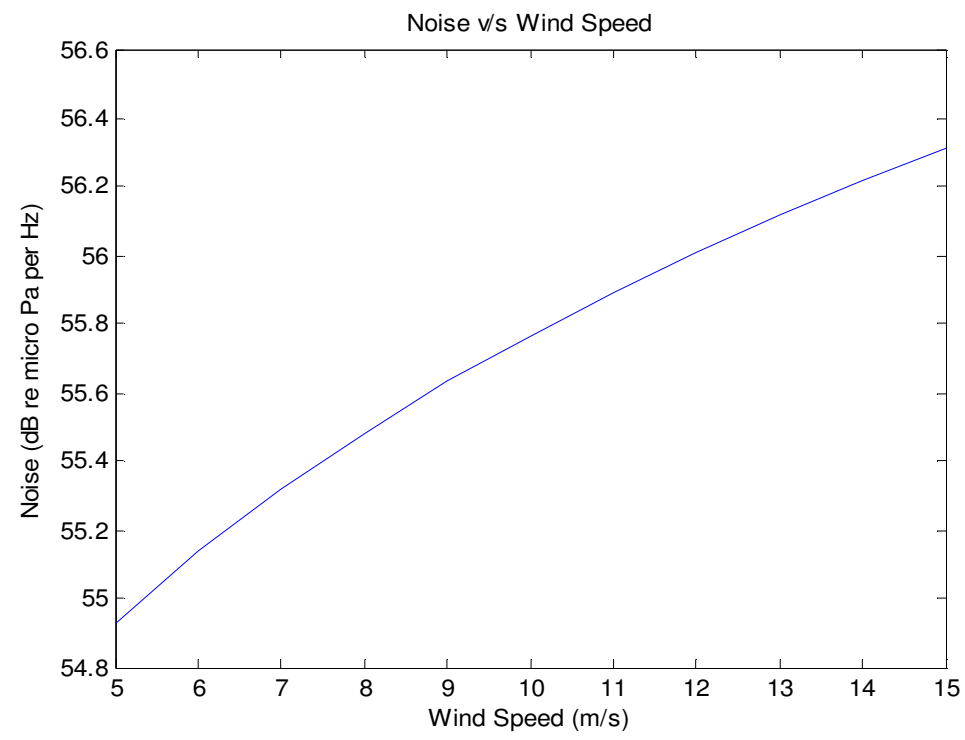

\subsection{Sound Speed}

Figure 9. Noise v/s wind speed

Using sound speed from equation 20, results have been plotted in the graphs shown in Figures 10, 11 and 12. A table 1 show that as the depth of a water body is varied from 0 meters to 1500 meters, the temperature and salinity decreases [17]. 
Table 1. Sound Speed with varying depth, temperature and salinity

\begin{tabular}{|c|c|c|c|c|}
\hline S.No. & $\begin{array}{c}\text { Depth (D) } \\
\text { in meters }\end{array}$ & $\begin{array}{c}\text { Temperature (T) in } \\
\text { degree Celsius }\end{array}$ & Salinity (S) in ppt & $\begin{array}{l}\text { Sound Speed } \\
\text { in } \mathbf{~ m} / \mathbf{s}\end{array}$ \\
\hline 1 & 0 & 18 & 0.03745 & 1475 \\
\hline 2 & 50 & 15 & 0.03602 & 1466 \\
\hline 3 & 100 & 10 & 0.03534 & 1448 \\
\hline 4 & 500 & 8 & 0.03511 & 1447 \\
\hline 5 & 1000 & 6 & 0.03490 & 1446 \\
\hline 6 & 1500 & 4 & 0.03405 & 1446 \\
\hline
\end{tabular}

Figure 10 and Figure 11 represent how temperature and salinity changes with depth respectively.

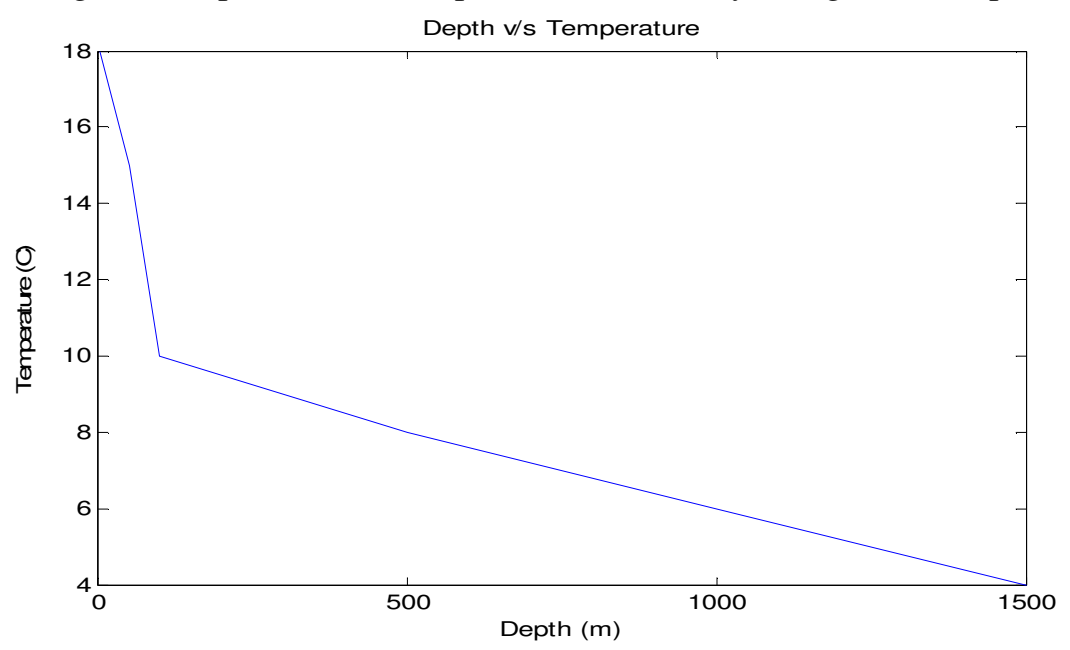

Figure 10. Depth v/s Temperature

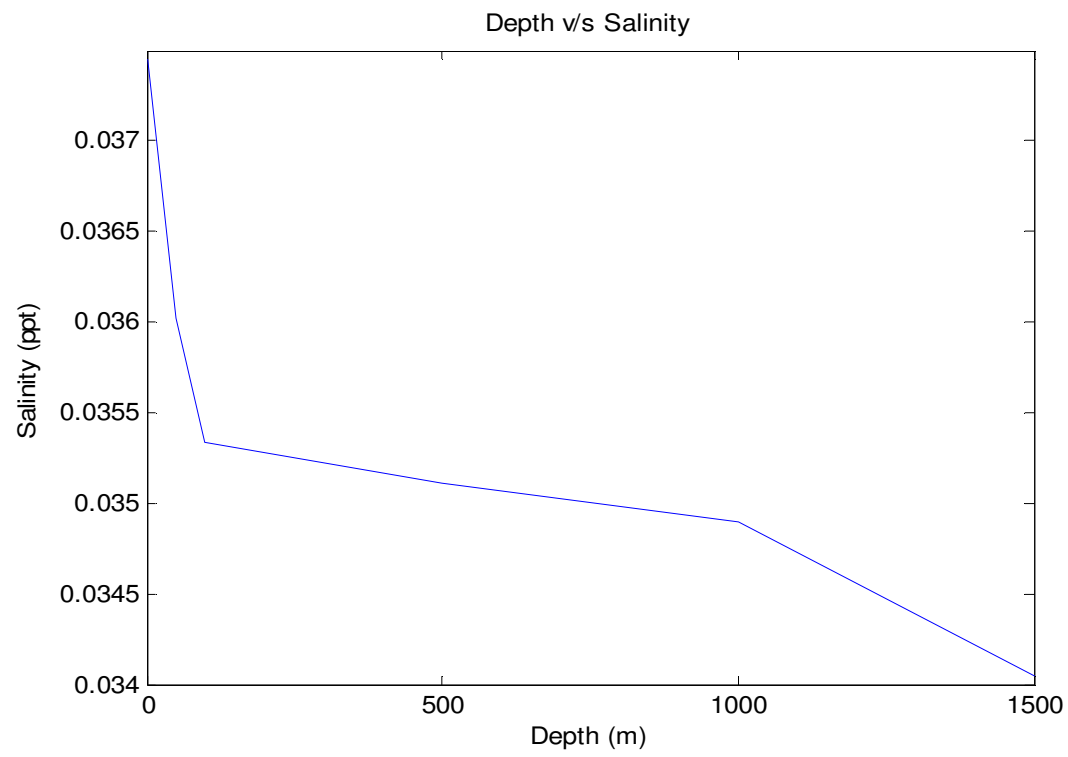

Figure 11. Depth v/s Salinity 
Figure 12 shows the relation between sound speed and depth with varying depth from $0 \mathrm{~m}$ to $1500 \mathrm{~m}$, varying temperature from $18^{\circ} \mathrm{C}$ to $4^{\circ} \mathrm{C}$ and varying salinity from $0.03745 \mathrm{ppt}$ to 0.03405 . It may be observed from the results that initially the change in the speed is very large but at greater depths the variation is very low.

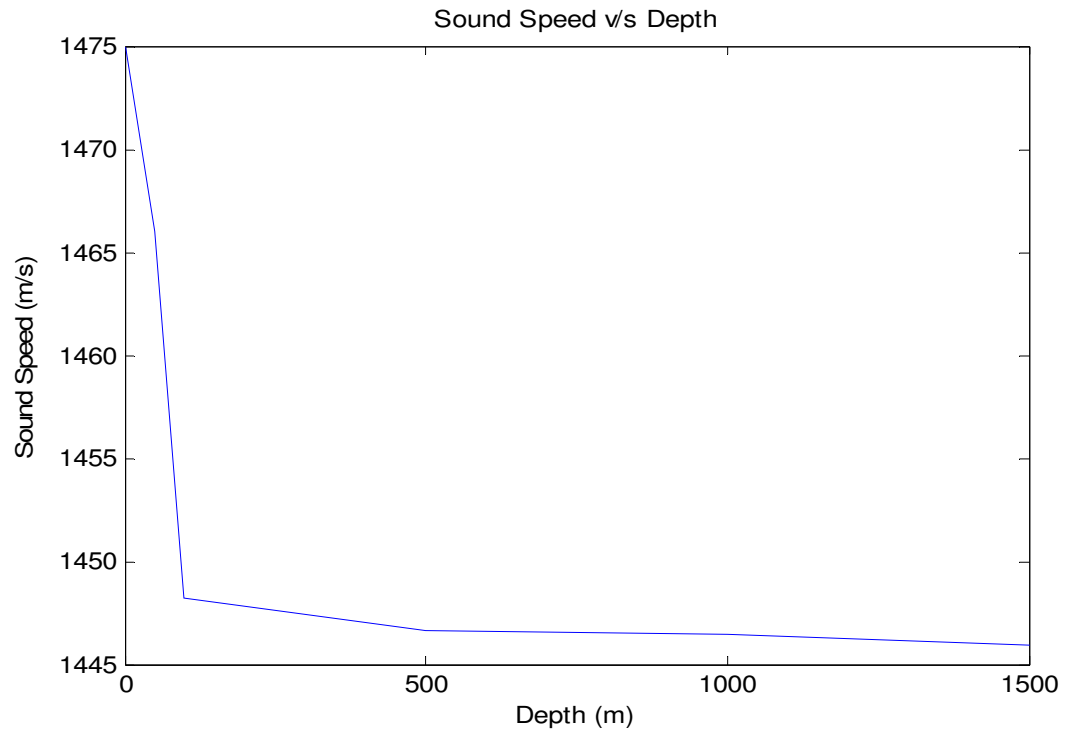

Figure 12. Sound Speed v/s Depth

\section{Conclusion}

The paper analyses the different acoustic channel characteristics to be considered for communication in underwater wireless sensor networks. The first being the attenuation profiles like Thorp model, Fisher and Simmons model and Ainslie \& McColm model have been studied. From the simulation of these attenuation models, it has been observed that the attenuation coefficient increases with the increasing frequency in water. Moreover, the noise in underwater wireless sensor networks has also been studied, depending upon the parameters like wind speed, shipping factor, concluding that the noise also increases with increasing frequency. Another characteristic observed is the sound speed, which depends upon the depth, temperature and salinity of the sea or ocean. The speed of acoustic signal decreases with increased depth.

\section{REFERENCES}

[1] Y.A.Malkani, A.Keerio, J.A.Mahar, G.A.Memon \& H. Keeriom, (2012) "Localization, Routing and Data Gathering in Wireless Sensor Networks (WSNs)", Sindh Univ. Res. Jour. (Sci. Ser.), Vol. 44, pp15-22.

[2] Ayaz, Muhammad, Imran Baig, Azween Abdullah \& Ibrahima Faye, (2011) "A survey on routing techniques in underwater wireless sensor networks", Journal of Network and Computer Applications, Elsevier publisher, Vol. 34, No. 6, pp1908-1927.

[3] Akyildiz, Ian F., Dario Pompili \& Tommaso Melodia, (2005) "Underwater acoustic sensor networks: research challenges", Ad hoc networks, Elsevier publisher, Vol. 3, No.3, pp257-279.

[4] Akyildiz, Ian F., Dario Pompili, and Tommaso Melodia, (2006) "State-of-the-art in protocol research for underwater acoustic sensor networks", Proceedings of the 1st ACM international workshop on Underwater networks. ACM. 
[5] Akyildiz, Ian F., Dario Pompili \& Tommaso Melodia, (2004) "Challenges for efficient communication in underwater acoustic sensor networks", ACM Sigbed Review, Vol. 1, No. 2, pp3-8.

[6] Prasan, U. Devee \& S. Murugappan, (2012) "Underwater Sensor Networks: Architecture, Research Challenges and Potential Applications", International Journal of Engineering Research and Applications, Vol. 2, No. 2, pp251-256.

[7] Guide, M. U. S. (1998). The mathworks. Inc., Natick, MA, 5, 333.

[8] Thorp, William H., (1967) "Analytic Description of the Low-Frequency Attenuation Coefficient", The Journal of the Acoustical Society of America, Vol. 42, No. 1, pp270-270.

[9] F. H. Fisher, V. P. Simmons, (1977) "Sound Absorption in Sea Water", The Journal of the Acoustical Society of America, Vol. 62, No. 3, pp558-564.

[10] Ainslie, Michael A. \& James G. McColm, (1998) "A simplified formula for viscous and chemical absorption in sea water", The Journal of the Acoustical Society of America, Vol. 103, No. 3, pp1671-1672.

[11] Harris III, Albert F. \& Michele Zorzi, (2007) "Modeling the underwater acoustic channel in ns2", Proceedings of the 2nd international conference on Performance evaluation methodologies and tools.

[12] J. Llor, E. Torres \& P. Garrido, (2009) "Analyzing the behavior of acoustic link models in underwater wireless sensor networks", Proceedings of the 4th ACM workshop on Performance monitoring and measurement of heterogeneous wireless and wired networks, pp9-16.

[13] Mackenzie, Kenneth V, (1981) "Nine-term equation for sound speed in the oceans", The Journal of the Acoustical Society of America, Vol. 70, No.3, pp807-812.

[14] Llor, Jesús, and Manuel P. Malumbres, (2010) "Modelling Underwater Wireless Sensor Networks", Intech open, pp1-20.

[15] Sehgal, Anuj, (2013) "Analysis \& Simulation of the Deep Sea Acoustic Channel for Sensor Networks", Lulu. Com.

[16] Jing, Nan, Weihong Bi \& Qing Yue, (2011) "Attack simulation model and channel statistics in underwater acoustic sensor networks", Tsinghua Science \& Technology, Vol. 16, No. 6, pp611621.

[17] Sheena Kohli \& Partha Pratim Bhattacharya, (2015, in press) "Characterization of Acoustic Channel for Underwater Wireless Sensor Networks", $12^{\text {th }}$ IEEE India International conference INDICON $\left(E^{3}-C^{3}\right)$, New Delhi.

\section{AUTHORS}

Yamini Kularia was born in India on January 30, 1992. She received her B.Tech degree in Electronics and Communication Engineering from Jagannath Gupta Institute of Engineering and Technology, Rajasthan Technical University, India in 2012 and currently is a M. Tech (Wireless Communication \& Technology) student in Mody University of Science and Technology, Lakshmangarh, Rajasthan, India.

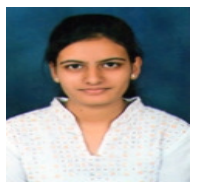

Sheena Kohli is currently working as Assistant Professor in Department of Computer Science and Engineering at Mody University of Science and Technology, Lakshmangarh, Rajasthan, India. She has received her B.Tech degree in Information Technology from Rajasthan Technical University, in 2010. She completed her M.Tech in Information Technology from Banasthali University, Rajasthan, India, in 2012. Her research interests include wireless sensor networks and underwater acoustic sensor networks.

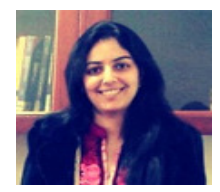


Dr. Partha Pratim Bhattacharya was born in India on January 3, 1971. He has 19 years of experience in teaching and research. He served many reputed educational Institutes in India in various positions starting from Lecturer to Professor and Principal. At present he is working as Professor in Department of Electronics and Communication Engineering in the Faculty of Engineering and Technology, Mody University of Science and Technology, Lakshmangarh, Rajasthan, India. He worked on Microwave devices and systems and

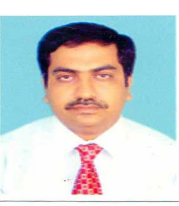
mobile cellular communication systems. He has published a good number of papers in refereed journals and conferences. His present research interest includes wireless communication. Dr. Bhattacharya is a member of The Institution of Electronics and Telecommunication Engineers, India and The Institution of Engineers, India. He is the recipient of Young Scientist Award from International Union of Radio Science in 2005. He is working as the chief editor, editorial board member and reviewer in many reputed journals. 